\title{
The Needfinding Machine
}

\author{
Nikolas Martelaro \& Wendy Ju \\ Stanford University \\ Stanford, CA \\ \{nikmart, wendyju\}@stanford.edu
}

\begin{abstract}
Robotics and automation technologies have begun to extend ubiquitous computing to include interactive objects with physical and social agency. My thesis, the Needfinding Machine, explores using social robots as design tools for understanding people and creating rich adaptive user experiences. In essence, the Needfinding Machine enables a conversation between a designer and a person through a social robot. This conversation can support designers during needfinding and enable future social robots to elicit meaningful information from users.
\end{abstract}

\section{CCS Concepts}

•Human-centered computing $\rightarrow$ User centered design;

\section{Keywords}

Interaction design; Needfinding; Human-robot interaction

\section{INTRODUCTION}

Robotics and automation technologies have begun to extend the vision of ubiquitous computing [13] into an era of ubiquitous robotics. Interactive objects with both physical and social agency, such as the Amazon Echo, are now widely available social robots. This agency, predicated by the social attributes people ascribe technology [8], allows more meaningful and conversational interaction to occur. Human-computer interaction and human-robot interaction designers often leverage these highly social interactions to elicit personal and meaningful information from people. For example, companion robots such as Paro have been shown to elicit meaningful conversation from elders and suggest new directions for social robot design $[1,12]$. These interactions provide insights into both user behavior and extend further to their personal needs and values.

My thesis is called "The Needfinding Machine" and defines an interaction design methodology that allows designers to

Permission to make digital or hard copies of part or all of this work for personal or classroom use is granted without fee provided that copies are not made or distributed for profit or commercial advantage and that copies bear this notice and the full citation on the first page. Copyrights for third-party components of this work must be honored For all other uses, contact the owner/author(s).

HRI '17 Companion March 06-09, 2017, Vienna, Austria

(C) 2017 Copyright held by the owner/author(s).

ACM ISBN 978-1-4503-4885-0/17/03

DOI: http://dx.doi.org/10.1145/3029798.3034811 interact through socially-enabled devices that can elicit meaningful interactions, monitor sensor data, record interactions, and converse with users in their real-time context. This provides conversational, closed-loop feedback to designers throughout the usage of the product. This can support designers to conduct qualitative needfinding research. While the needs themselves do not lead a designer to a direct solution, they can help to reframe problems and build a richer understanding of what is important to the user [9]. My primary research question is how can designers create and use robotic objects that elicit meaningful interaction to better understand people and improve the design of their products? The primary contributions of my research are design strategies for creating interactive "needfinding machines" (elicitation techniques, social behaviors, and physical attributes) and an understanding of how designers can engage with people through these interactive objects. This understanding can then inform both the design process for socially enabled objects and help generate potential designs for socially adaptable robots.

\section{RESEARCH METHODOLOGY}

The vision of the Needfinding Machine takes inspiration from cultural [3] and technology [4] probes as a method to support designers exploring user needs and generating new interaction concepts. The Needfinding Machine builds upon technology probes by directly linking designers to users through a product and allowing the designer to act in a situated manner where the overall goals of interaction are known but the specific behaviors are adaptable and rely on understanding previous interactions [11].

I approach my work using a research through design methodology [14], designing and reflecting upon the development of social robots. Functionally, these designs build upon Wizard-Of-Oz experimentation techniques [2] that have often been used in HCI and HRI research [10] to simulate technology that is currently not available and to help explore possible interaction strategies for future algorithms. The Needfinding Machine presents and alternative use of Wizard-of-Oz, framing its use for the purposes of needfinding through designer-in-the-loop dialogue with the user. My work utilizes experimental studies testing interaction strategies and robot design features that can elicit meaningful conversation between a person and a robot. Additionally, I conduct observational studies of designers using needfinding machines, exploring how they engage with users and how these interactions influence their design process. 


\section{PREVIOUS WORK}

An early study I conducted using a robot to teach students electronics lead me to the idea of robots eliciting disclosure as a means of needfinding. In an attempt to improve student learning, I designed a robot that would be interested in the student and found that students told personal stories in response to questions from the robot [5]. Although not always related to the task, the student's personal stories helped me empathize with them and supported my thinking about new ways to design education support tools. Building upon this first study, I then explored specific robot attributes, namely, vulnerability and expressivity, and hypothesized that these social features would impact trust, disclosure, and companionship with the robot tutor. The results of this study suggest that both vulnerability and expressivity aid in eliciting disclosure and trust from people, but that these attributes work in different ways, with vulnerability eliciting more trust and expressivity eliciting more disclosure [7]. I have used the work from these studies to inform the design of needfinding machines to elicit meaningful interaction between the user and robot, ultimately providing the designer with information relevant to needfinding.

These strategies for designing needfinding machines have now extended beyond the lab and onto the open road. As selfdriving cars become a reality and designers begin to create the interaction between the driver and autonomous systems, I am exploring how the Needfinding Machine can support designers in understanding the needs of future drivers. To do this, I have built and tested a system to connect automotive designers to remote drivers. This system provides live video, audio, car data, and Wizard-of-Oz speech and physical interfaces to enable remote observation and interaction prototyping on the road [6]. The system integrates environmental (road-context, location), system level (speed, braking, cruise control status, etc.), and social information (user facing video, elicited speech) to let the designer explore what the driver is doing and why they are doing it. The findings from this deployment illustrate how designers use this remote interaction through the car as both a design research and a design prototyping tool. Designers were able to conduct naturalistic observations, contextual inquiry, and responsive interaction prototyping. The designers learned not only how the driver reacted to and felt about the autonomous systems, but also began to understand what needs were unmet and what needs could arise from the use of these systems. These needs often centered around understanding how to enable certain features and how to understand what decisions the car was making. The designers are now using these methods to explore and design interfaces that allow the car to elicit the driver's understanding and educate the driver about its own features.

\section{REMAINING WORK}

In my remaining work, I am exploring robotic agents for automated driving assistance and machine learning based music recommendation. I am co-developing needfinding machines with professional designers and conducting observational studies to explore how these designers use the methodology to understand their user's needs and interactively prototype new social interfaces for the underlying autonomous systems. This work will also explore the limitations of the Needfinding Machine, such as the challenges of conducting real-time interaction, potential privacy concerns that may bias user interactions, and the abilities for such methods to support professional design work at scale.

Through this method, designers can more deeply understand their users and create new interactions with autonomy that are sensitive to people's needs and values. In turn, this can foster future development of autonomous systems that are more socially aware and adaptive.

\section{ACKNOWLEDGMENTS}

This work is supported by the National Science Foundation GRFP Grant \#DGE-114747.

\section{REFERENCES}

[1] W.-L. Chang and S. Šabanović. Interaction Expands Function: Social Shaping of the Therapeutic Robot PARO in a Nursing Home. In HRI '15, pages 343-350, New York, NY, USA, 2015. ACM.

[2] N. Dahlbäck, A. Jönsson, and L. Ahrenberg. Wizard of Oz Studies: Why and How. In IUI '93, pages 193-200, New York, NY, USA, 1993. ACM.

[3] B. Gaver, T. Dunne, and E. Pacenti. Design: Cultural probes. interactions, 6(1):21-29, 1999.

[4] H. Hutchinson, W. Mackay, B. Westerlund, B. B. Bederson, A. Druin, C. Plaisant, M. Beaudouin-Lafon, S. Conversy, H. Evans, and H. Hansen. Technology probes: Inspiring design for and with families. In $\mathrm{CHI}$ '03, pages 17-24. ACM, 2003.

[5] M. F. Jung, N. Martelaro, H. Hoster, and C. Nass. Participatory materials: Having a reflective conversation with an artifact in the making. In DIS '14, pages 25-34. ACM, 2014.

[6] N. Martelaro and W. Ju. WoZ Way: Enabling real-time interaction prototyping and on-road observation. In CSCW' '17, Portland, OR, 2017.

[7] N. Martelaro, V. C. Nneji, W. Ju, and P. Hinds. Tell Me More: Designing HRI to Encourage More Trust, Disclosure, and Companionship. In $H R I$ '16, pages 181-188, Piscataway, NJ, USA, 2016. IEEE Press.

[8] C. Nass, J. Steuer, and E. R. Tauber. Computers Are Social Actors. In CHI'94, pages 72-78, New York, NY, USA, 1994. ACM.

[9] D. Patnaik and R. Becker. Needfinding: The Why and How of Uncovering People's Needs. Design Management Journal, 10(2):37-43, Apr. 1999.

[10] L. D. Riek. Wizard of oz studies in hri: A systematic review and new reporting guidelines. Journal of Human-robot Interaction, 1(1), 2012.

[11] L. A. Suchman. Plans and Situated Actions: The Problem of Human-Machine Communication. Cambridge University Press, Nov. 1987.

[12] S. Turkle, W. Taggart, C. D. Kidd, and O. Dasté. Relational artifacts with children and elders: The complexities of cybercompanionship. Connection Science, 18(4):347-361, Dec. 2006.

[13] M. Weiser. The computer for the 21st century. Scientific American, 265(3):94-104, 1991.

[14] J. Zimmerman, J. Forlizzi, and S. Evenson. Research through design as a method for interaction design research in $\mathrm{HCI}$. In $\mathrm{CHI}$ 'O\%, pages 493-502. ACM, 2007. 